\title{
Local well-posedness for the periodic higher order KdV type equations
}

\author{
Hiroyuki Hirayama
}

\begin{abstract}
Higher order $\mathrm{KdV}$ type equations are the equation replaced by a higher order derivative $\partial_{x}^{2 k+1}$ for the KdV equation. Recently, the local well-posedness result for these equations on torus have been given by Gorsky and Himonas (Math. Comput. Simul. 80:173-183, 2009). We extend this result by improving a bilinear estimate used in the Fourier restriction norm method.
\end{abstract}

Mathematics Subject Classification (2000). 35Q53, 35G25.

Keywords. KdV equation, Well-posedness, Cauchy problem, Fourier restriction norm.

\section{Introduction}

We consider the Cauchy problem of the periodic high order KdV type equations;

$$
\begin{cases}\partial_{t} u+(-1)^{k+1} \partial_{x}^{2 k+1} u+\frac{1}{2} \partial_{x}\left(u^{2}\right)=0, & (t, x) \in[0, T] \times \mathbb{T} \\ u(0, x)=u_{0}(x), & u_{0} \in H^{s}(\mathbb{T})\end{cases}
$$

where $k \in \mathbb{N}$ and the unknown function $u$ is real valued. The aim of this paper is to prove the local well-posedness (LWP for short) of (1.1) with low regularity initial data.

When $k=1$, the Eq. (1.1) is called "KdV equation". We first introduce some known results for the KdV equation. Bourgain [2] introduced a new method called "Fourier restriction norm method" and proved that the KdV equation is LWP in $L^{2}(\mathbb{T})$. Kenig et al. [17] refined a bilinear estimate used in the Fourier restriction norm method, and proved that the KdV equation is LWP in $H^{s}(\mathbb{T})$ for $s \geq-1 / 2$. By using the local well-posedness result and the almost conservation low, Colliander et al. [5] obtained that the KdV equation is the globally well-posed in $H^{s}(\mathbb{T})$ for $s \geq-1 / 2$. Their method is called "I-method". On the other hand, Christ et al. [4] proved that the KdV equation 
is ill-posed. More precisely, the data-to-solution map is not uniformly continuous on $H^{s}(\mathbb{T})$ for $-2<s<-1 / 2$. LWP of the non-periodic KdV equation also was studied by many people before Bourgain's work $[1,7,11,13,14]$ and after Bourgain's work [5, 10,15, 17-19,21,24].

Next, we introduce some known results for the fifth order KdV type equations

$$
\partial_{t} u+\alpha \partial_{x}^{5} u+\beta \partial_{x}^{3} u+\partial_{x}\left(u^{2}\right)=0
$$

and

$$
\partial_{t} u-\partial_{x}^{5} u-30 u^{2} \partial_{x} u+20 \partial_{x} u \partial_{x}^{2} u+10 u \partial_{x}^{3} u=0 .
$$

Especially, (1.2) is called "Kawahara equation". LWP of these equations are studied for non-periodic case. For the known results of the non-periodic Kawahara equation, see $[3,6,12,25]$ and the Eq. (1.3), see [20,23].

We return to introduce the known result for (1.1) for general $k \in \mathbb{N}$. Recently, Gorsky and Himonas [9] have proved that (1.1) is LWP in $H^{s}(\mathbb{T})$ for $s \geq-1 / 2$ by an argument similar to [17]. The main result in the present paper is an extension of the result by Gorsky and Himonas.

Finally, we introduce the high order nonlinear dispersive equations related to (1.1). Kenig et al. [16] studied the high order nonlinear dispersive equations

$$
\partial_{t} u+\partial_{x}^{2 k+1} u+P\left(u, \partial_{x} u, \ldots, \partial_{x}^{2 k} u\right)=0,
$$

where $P$ is a polynomial without constant and linear terms. They proved that (1.4) is LWP in $L^{2}\left(|x|^{m} d x\right) \cap H^{s}(\mathbb{R})$, where $s>0$ and $m \in \mathbb{Z}^{+}$are sufficiently large. Pilod [22] proved that (1.4) with

$$
P\left(u, \partial_{x} u, \ldots, \partial_{x}^{2 k} u\right)=\sum_{0 \leq k_{1}+k_{2} \leq 2 k} a_{k_{1}, k_{2}} \partial_{x}^{k_{1}} u \partial_{x}^{k_{2}} u
$$

is LWP in $H^{s}(\mathbb{R}) \cap H^{s-2 k}\left(x^{2} d x\right)$ for $s \in \mathbb{N}$ and $s>2 k+1 / 4$. He also proved some ill-posed results for (1.4).

Without loss of generality, we can assume that $\widehat{u}_{0}(0)=0$ by the following transform:

$$
u \mapsto u-\frac{1}{2 \pi} \int_{\mathbb{T}} u_{0}(x) d x .
$$

Therefore, we have only to prove LWP of $(1.1)$ in $\widetilde{H}^{s}(\mathbb{T})$, where

$$
\widetilde{H}^{s}(\mathbb{T}):=\left\{f \in H^{s}(\mathbb{T}) \mid \widehat{f}(0)=0\right\}
$$

and $\widehat{u}$ is the Fourier transform of $u$ with respect to $x$. We note that $\widetilde{H}^{s}(\mathbb{T})$ is a Banach space with respect to the norm

$$
\|f\|_{\widetilde{H}^{s}(\mathbb{T})}:=\left.\left.|| n\right|^{s} \widehat{f}\right|_{L_{n}^{2}} .
$$

Next, we define the Bourgain spaces $Z^{s}(\lambda)$ and $Z_{[0, T]}^{s}(\lambda)$, where $\lambda$ is the scale parameter. We will use the scaling property (4.2) in the proof of Theorem 1.2. 
Definition 1.1. Let $\lambda \geq 1, \mathbb{T}_{\lambda}:=\mathbb{R} /(2 \pi \lambda) \mathbb{Z}$ and $X_{\lambda}$ be the space of all $F$ : $\mathbb{R} \times \mathbb{T}_{\lambda} \rightarrow \mathbb{R}$ such that $F(\cdot, x) \in \mathcal{S}(\mathbb{R})$ for all $x \in \mathbb{T}_{\lambda}$, the map $x \mapsto F(\cdot, x)$ is $C^{\infty}$ and $\widetilde{F}(\tau, 0)=0$ for all $\tau \in \mathbb{R}$, where $\mathcal{S}(\mathbb{R})$ is the Schwartz space and $\widetilde{F}$ is the Fourier transform of $F$ with respect to $x$ and $t$.

(1) For $s \in \mathbb{R}$, we define the function space $Z^{s}(\lambda)$ as the completion of $X_{\lambda}$ with respect to the norm

$$
\|u\|_{Z^{s}(\lambda)}:=\|u\|_{X^{s}(\lambda)}+\|u\|_{Y^{s}(\lambda)}
$$

where

$$
\|u\|_{X^{s}(\lambda)}:=\left\|\left.\left\langle\tau-n^{2 k+1}\right\rangle^{1 / 2}|n|^{s} \widetilde{u}\right|_{L_{n}^{2}(\lambda) L_{\tau}^{2}},\right\| u\left\|_{Y^{s}(\lambda)}:=\right\||n|^{s} \widetilde{u} \|_{L_{n}^{2}(\lambda) L_{\tau}^{1}} .
$$

(2) We define the function space $Z_{[0, T]}^{s}(\lambda)$ and the norm $\|\cdot\|_{Z_{[0, T]}^{s}(\lambda)}$ as

$$
\begin{gathered}
Z_{[0, T]}^{s}(\lambda):=\left\{\left.u\right|_{[0, T]} \mid u \in Z^{s}(\lambda)\right\} \\
\|u\|_{Z_{[0, T]}^{s}(\lambda)}:=\inf \left\{\|v\|_{Z^{s}(\lambda)} \mid v \in Z^{s}(\lambda), v(t)=u(t) \text { on }[0, T]\right\} .
\end{gathered}
$$

We omit to write down " $(\lambda)$ " when $\lambda=1$.

The main result in the present paper is the following theorem:

Theorem 1.2. Let $k \in \mathbb{N}$. If $s \geq-k / 2$ then (1.1) is $L W P$ in $H^{s}(\mathbb{T})$. More particularly, for all $r \geq 1$ and $u_{0} \in B_{r}\left(\widetilde{H}^{s}(\mathbb{T})\right)$, there exist $T=T(r)>0$ and a solution $u \in Z_{[0, T]}^{s} \cap C\left([0, T] ; \widetilde{H}^{s}(\mathbb{T})\right)$ of $(1.1)$. Such solution $u$ is unique in a closed subset of $Z_{[0, T]}^{s}$. Moreover, the map $u_{0} \mapsto u$ from $B_{r}\left(\widetilde{H}^{s}(\mathbb{T})\right)$ into $Z_{[0, T]}^{s}$ is Lipschitz continuous.

Bilinear estimate plays an important role to prove LWP of (1.1). Gorsky and Himonas derived the following bilinear estimate for $s \geq-1 / 2$;

$$
\left\|\mathcal{F}^{-1}\left[\left\langle\tau-n^{2 k+1}\right\rangle^{-1} \widehat{\partial_{x}(u v)}\right]\right\|_{Z^{s}} \leq C\|u\|_{Z^{s}}\|v\|_{Z^{s}} .
$$

But as mentioned in [9], the estimate (1.5) with $s<-1 / 2$ has been open problem. We extend (1.5) to prove Theorem 1.2 and obtain the following bilinear estimate;

Theorem 1.3. Let $k \in \mathbb{N}$ and $\lambda \geq 1$. For $s \geq-k / 2$, there exist a positive constant $C_{0}$ and $\epsilon$ satisfying $0<\epsilon<2 k+s-1 / 2$ such that the following bilinear estimate holds;

$$
\left\|\mathcal{F}^{-1}\left[\left\langle\tau-n^{2 k+1}\right\rangle^{-1} \widehat{\partial_{x}(u v)}\right]\right\|_{Z^{s}(\lambda)} \leq C_{0} \lambda^{\epsilon}\|u\|_{Z^{s}(\lambda)}\|v\|_{Z^{s}(\lambda)},
$$

where $C_{0}$ does not depend on $\lambda$.

On the other hand, we also obtain negative result for $s<-k / 2$.

Theorem 1.4. Let $k \in \mathbb{N}$. For any $s<-k / 2$, the bilinear (1.6) with $\lambda=1$ fails.

Remark 1.5. By Theorem 1.3 and $1.4, s=-k / 2$ is optimal regularity for the bilinear estimate (1.6). But this does not imply ill-posedness of (1.1) for $s<-k / 2$. 
The bilinear estimate (3.2) below with $\lambda=1$ can be written

$$
\begin{gathered}
|||n|^{1-k / 2}\left\langle\tau-n^{2 k+1}\right\rangle^{-1 / 2} \widetilde{u} * \widetilde{v} \|_{L_{n}^{2} L_{\tau}^{2}} \\
\left.\lesssim|||n|^{-k / 2}\left\langle\tau-n^{2 k+1}\right\rangle^{1 / 2} \widetilde{u}\right|_{L_{n}^{2} L_{\tau}^{2}}|||n|^{-k / 2}\left\langle\tau-n^{2 k+1}\right\rangle^{1 / 2} \widetilde{v} \|_{L_{n}^{2} L_{\tau}^{2}},
\end{gathered}
$$

where

$$
\widetilde{u} * \widetilde{v}(\tau, n)=\frac{1}{2 \pi} \sum_{n=n_{1}+n_{2}} \int_{\tau=\tau_{1}+\tau_{2}} \widetilde{u}\left(\tau_{1}, n_{1}\right) \widetilde{v}\left(\tau_{2}, n_{2}\right) d \tau_{1} .
$$

We note that the most difficult region to prove this estimate is $\left|n_{1}\right| \sim\left|n_{2}\right| \gg$ $|n|$. Gorsky and Himonas used the estimate

$$
\left|n^{2 k+1}-n_{1}^{2 k+1}-n_{2}^{2 k+1}\right| \gtrsim\left|n n_{1} n_{2}\right| \cdot|n|^{2 k-2}
$$

to prove (1.5). On the other hand, we use the refined estimate

$$
\left|n^{2 k+1}-n_{1}^{2 k+1}-n_{2}^{2 k+1}\right| \sim\left|n n_{1} n_{2}\right| \max \left\{|n|,\left|n_{1}\right|,\left|n_{2}\right|\right\}^{2 k-2},
$$

which is better estimate than (1.7) in the region $\left|n_{1}\right| \sim\left|n_{2}\right| \gg|n|$. Because of such reason, we could improve the bilinear estimate.

We will prove the bilinear estimate and give a counterexample in section 3. The proof of Theorem 1.2 will be given in section 4 .

\section{Notation.}

- We put $\langle\cdot\rangle:=1+|\cdot|$.

- We put $\mathbb{Z}^{*}:=\mathbb{Z} \backslash\{0\}$.

- For $\lambda \geq 1$, we put $\mathbb{Z}_{\lambda}:=\{n / \lambda \mid n \in \mathbb{Z}\}$ and $\mathbb{Z}_{\lambda}^{*}:=\mathbb{Z}_{\lambda} \backslash\{0\}$.

- For a function $f$ defined on $\mathbb{T}_{\lambda}$, we put

$$
\int_{\mathbb{T}_{\lambda}} f(x) d x:=\int_{0}^{2 \pi \lambda} f(x) d x .
$$

- For a function $f$ defined on $\mathbb{Z}_{\lambda}^{*}$, we put

$$
\int_{\mathbb{Z}_{\lambda}^{*}} f(n) d n:=\frac{1}{2 \pi \lambda} \sum_{n \in \mathbb{Z}_{\lambda}^{*}} f(n) .
$$

- For a function $f$ defined on $\mathbb{Z}_{\lambda}^{*}$, we put

$$
\|f\|_{L_{n}^{2}(\lambda)}:=\left(\int_{\mathbb{Z}_{\lambda}^{*}}|f(n)|^{2} d n\right)^{1 / 2} .
$$

- We define the Fourier transform of $f$ with respect to $x \in \mathbb{T}_{\lambda}$ as

$$
\mathcal{F}_{x}[f](n)=\widehat{f}(n):=\int_{\mathbb{T}_{\lambda}} f(x) e^{-i n x} d x\left(n \in \mathbb{Z}_{\lambda}\right) .
$$

- We define the Fourier transform of $F$ with respect to $(t, x) \in \mathbb{R} \times \mathbb{T}_{\lambda}$ as

$$
\mathcal{F}[F](\tau, n)=\widetilde{F}(\tau, n):=\int_{\mathbb{T}_{\lambda}} \int_{\mathbb{R}} F(t, x) e^{-i \tau t} e^{-i n x} d t d x .
$$

- For functions $F$ and $G$ defined on $\mathbb{R} \times \mathbb{Z}_{\lambda}^{*}$, we put

$$
F * G(\tau, n):=\int_{\mathbb{Z}_{\lambda}^{*} \backslash\{n\}} \int_{\mathbb{R}} F\left(\tau-\tau_{1}, n-n_{1}\right) G\left(\tau_{1}, n_{1}\right) d \tau_{1} d n_{1}
$$


- For a Banach space $X$ and $r>0$, we put

$$
B_{r}(X):=\left\{f \in X \mid\|f\|_{X} \leq r\right\} .
$$

- $A \lesssim B$ denotes $A \leq C B$ for some positive constant $C$ which does not depend on $\lambda$ and $A \sim B$ means $A \lesssim B$ and $B \lesssim A$.

\section{Preliminary}

In this section, we prepare to prove the bilinear estimate.

Lemma 2.1. Let $k \in \mathbb{N}$. If $p, q, r \in \mathbb{R}$ satisfy $p+q+r=0$ and $p^{2 k+1}+q^{2 k+1}+$ $r^{2 k+1}=0$ then at least one of $p, q$ and $r$ is equal to 0 .

Proof. We can assume $|p| \geq|q| \geq|r|$ and $p \geq 0, q \leq 0, r \leq 0$ without loss of generality. Since

$p^{2 k+1}=-\left(q^{2 k+1}+r^{2 k+1}\right)=-(q+r) \sum_{j=0}^{2 k} q^{2 k-j}(-r)^{j}=p \sum_{j=0}^{2 k}(-1)^{j}(-q)^{2 k-j}(-r)^{j}$

and

$$
p^{2 k+1}=p(-q-r)^{2 k}=p \sum_{j=0}^{2 k}\left(\begin{array}{c}
2 k \\
j
\end{array}\right)(-q)^{2 k-j}(-r)^{j}
$$

we have

$$
\operatorname{pqr} \sum_{j=1}^{2 k-1}\left\{\left(\begin{array}{c}
2 k \\
j
\end{array}\right)-(-1)^{j}\right\}(-q)^{2 k-1-j}(-r)^{j-1}=0 .
$$

While if $p q r \neq 0$, then we have

$$
\operatorname{pqr} \sum_{j=1}^{2 k-1}\left\{\left(\begin{array}{c}
2 k \\
j
\end{array}\right)-(-1)^{j}\right\}(-q)^{2 k-1-j}(-r)^{j-1}>0
$$

since $p \geq 0,-q \geq 0,-r \geq 0$ and

$$
\left(\begin{array}{c}
2 k \\
j
\end{array}\right)-(-1)^{j}>0
$$

for $1 \leq j \leq 2 k-1$. This contradicts the Eq. (2.1). Therefore we obtain $p q r=0$.

Nobu Kishimoto pointed out Lemma 2.2 and a proof of it to the author. The proof of Lemma 2.2 below is simpler than his.

Lemma 2.2. If $p, q, r \in \mathbb{R} \backslash\{0\}$ satisfy $p+q+r=0$ then

$$
\left|p^{2 k+1}+q^{2 k+1}+r^{2 k+1}\right| \sim|p q r| \max \{|p|,|q|,|r|\}^{2 k-2} .
$$


Proof. We can assume $|p| \geq|q| \geq|r|$ without loss of generality. By an elementary calculation, we have

$$
\begin{aligned}
p^{2 k+1}+q^{2 k+1}+r^{2 k+1} & =p^{2 k+1}+q^{2 k+1}-(p+q)^{2 k+1} \\
& =-\sum_{j=1}^{2 k}\left(\begin{array}{c}
2 k+1 \\
j
\end{array}\right) p^{2 k+1-j} q^{j} \\
& =-p q(p+q) \sum_{j=1}^{2 k-1}\left(\sum_{l=1}^{j}(-1)^{j+l}\left(\begin{array}{c}
2 k+1 \\
l
\end{array}\right)\right) p^{2 k-1-j} q^{j-1} \\
& =p q r \cdot p^{2 k-2} Q(\beta),
\end{aligned}
$$

where

$$
Q(\beta)=\sum_{j=1}^{2 k-1} \alpha_{j} \beta^{j-1}, \quad \alpha_{j}:=\sum_{l=1}^{j}(-1)^{j+l}\left(\begin{array}{c}
2 k+1 \\
l
\end{array}\right), \quad \beta:=\frac{q}{p} .
$$

We note that $-1<\beta \leq-1 / 2$ and $Q(-1)=2 k+1$. Furthermore, we have

$$
|Q(\beta)|=\frac{\left|p^{2 k+1}+q^{2 k+1}+r^{2 k+1}\right|}{|p q r| \cdot|p|^{2 k-2}} \neq 0
$$

for $-1<\beta \leq-1 / 2$ from Lemma 2.1. This implies

$$
\inf _{-1<\beta \leq-1 / 2}|Q(\beta)|>0 \text {. }
$$

Therefore, we obtain

$$
\left|p^{2 k+1}+q^{2 k+1}+r^{2 k+1}\right|=|p q r| \cdot|p|^{2 k-2}|Q(\beta)| \sim|p q r| \max \{|p|,|q|,|r|\}^{2 k-2} \text {. }
$$

Lemma 2.3. If $l, m, n \in \mathbb{Z}_{\lambda}^{*}$ satisfy $l+m+n=0$, then we have

$$
\left|l^{2 k+1}+m^{2 k+1}+n^{2 k+1}\right| \gtrsim \frac{1}{\lambda} \max \{|l|,|m|,|n|\}^{2 k} .
$$

Proof. We can assume $|l| \geq|m| \geq|n|$ without loss of generality. Since

$$
|l| \leq|m|+|n| \leq 2|m|
$$

and

$$
|n| \geq \frac{1}{\lambda}
$$

we obtain

$$
|l m n| \geq \frac{1}{2 \lambda}|l|^{2} \sim \frac{1}{\lambda} \max \{|l|,|m|,|n|\}^{2} .
$$

Therefore, we have (2.2) by Lemma 2.2 .

Lemma 2.4. Let $a \in \mathbb{R}$. For any $\delta$ and $\delta^{\prime}$ satisfying $0 \leq \delta^{\prime}<\delta<1$, we have

$$
\int_{\mathbb{R}} \frac{1}{\langle\theta\rangle\langle\theta+a\rangle^{1-\delta^{\prime}}} d \theta \lesssim \frac{1}{\langle a\rangle^{\delta-\delta^{\prime}}},
$$

where the implicit constant depends only on $\delta$ and $\delta^{\prime}$.

For the proof of Lemma 2.4, see Lemma 4.2 in [8]. 
Lemma 2.5. Let $\lambda \geq 1$. For any $\delta$ satisfying $1 / m<\delta$, we have

$$
\int_{\mathbb{Z}_{\lambda}^{*}} \frac{1}{\left\langle\lambda^{-1} P(n)\right\rangle^{\delta}} d n \lesssim \lambda^{\delta(m+1)-1},
$$

where $P(x)$ is a polynomial of the form

$$
P(x):=\sum_{j=0}^{m} c_{j} x^{j}
$$

with $c_{0}, \ldots, c_{m} \in \mathbb{R}, c_{m}$ does not depend on $\lambda$ and the implicit constant depends only on $c_{m}$.

Proof. Let $\gamma_{1}, \ldots, \gamma_{m} \in \mathbb{C}$ be the roots of the equation

$$
P(x)=0 .
$$

Then we have

$$
P(n)=c_{m} \prod_{j=1}^{m}\left(n-\gamma_{j}\right)
$$

We put

$$
C_{j}:=\left\{n \in \mathbb{Z}_{\lambda}^{*}|| n-\gamma_{j} \mid \leq 1\right\}, \quad C:=\bigcup_{j=1}^{m} C_{j}, D:=\mathbb{Z}_{\lambda}^{*} \backslash C .
$$

Since $\# C_{j} \lesssim \lambda$, we have

$$
\int_{C} \frac{1}{\left\langle\lambda^{-1} P(n)\right\rangle^{\delta}} d n \leq \int_{C} 1 d n=\frac{1}{2 \pi \lambda} \# C \leq \frac{1}{2 \pi \lambda} \sum_{j=1}^{m} \# C_{j} \lesssim 1 .
$$

On the other hand, since

$$
1 \leq\left|n-\gamma_{j}\right|
$$

for any $n \in D$ and $1 \leq j \leq m$, we have

$$
\prod_{j=1}^{m}\left\langle n-\gamma_{j}\right\rangle \sim \prod_{j=1}^{m}\left|n-\gamma_{j}\right| \leq \lambda\left\langle\lambda^{-1} \prod_{j=1}^{m}\left(n-\gamma_{j}\right)\right\rangle .
$$

Therefore, we have

$$
\int_{D} \frac{1}{\left\langle\lambda^{-1} P(n)\right\rangle^{\delta}} d n \lesssim \lambda^{\delta} \int_{D} \frac{1}{\prod_{j=1}^{m}\left\langle n-\gamma_{j}\right\rangle^{\delta}} d n \leq \lambda^{\delta} \prod_{j=1}^{m}\left(\int_{D} \frac{1}{\left\langle n-\gamma_{j}\right\rangle^{\delta m}} d n\right)^{1 / m}
$$

by Holder's inequality. Since

$$
\int_{D} \frac{1}{\left\langle n-\gamma_{j}\right\rangle^{\delta m}} d n \leq \frac{1}{2 \pi \lambda} \sum_{n \in \mathbb{Z}^{*}} \frac{\lambda^{\delta m}}{\left\langle n-\lambda \gamma_{j}\right\rangle^{\delta m}} \lesssim \lambda^{\delta m-1}
$$

by $1 / m<\delta$, we obtain

$$
\int_{D} \frac{1}{\left\langle\lambda^{-1} P(n)\right\rangle^{\delta}} d n \lesssim \lambda^{\delta(m+1)-1}
$$




\section{Proof of the bilinear estimate}

In this section, we give the proofs of Theorem 1.3 and Theorem 1.4.

Proof of Theorem 1.3. For $s>-k / 2$, we note that

$$
\begin{aligned}
\left|n^{s}(\widetilde{u} * \widetilde{v})\right| & \lesssim|n|^{-k / 2}\left(\left|\left(n^{s+k / 2} \widetilde{u}\right) * \widetilde{v}\right|+\left|\widetilde{u} *\left(n^{s+k / 2} \widetilde{v}\right)\right|\right) \\
& \lesssim \lambda^{s+k / 2}|n|^{-k / 2}\left|\left(n^{s+k / 2} \widetilde{u}\right) *\left(n^{s+k / 2} \widetilde{v}\right)\right|
\end{aligned}
$$

by the triangle inequality and $\lambda^{-1} \leq|n|$ for all $n \in \mathbb{Z}_{\lambda}^{*}$. Thus, we only need to prove the bilinear estimate (1.6) for the case $s=-k / 2$. We put

$\widetilde{f}(\tau, n):=\left\langle\tau-n^{2 k+1}\right\rangle^{1 / 2}|n|^{-k / 2} \widetilde{u}(\tau, n), \quad \widetilde{g}(\tau, n):=\left\langle\tau-n^{2 k+1}\right\rangle^{1 / 2}|n|^{-k / 2} \widetilde{v}(\tau, n)$,

then we have

$$
\|u\|_{X^{-k / 2}(\lambda)}=\|\widetilde{f}\|_{L_{n}^{2}(\lambda) L_{\tau}^{2}},\|v\|_{X^{-k / 2}(\lambda)}=\|\widetilde{g}\|_{L_{n}^{2}(\lambda) L_{\tau}^{2}} .
$$

Furthermore, we put

$n_{2}:=n-n_{1}, \tau_{2}:=\tau-\tau_{1}, \sigma:=\tau-n^{2 k+1}, \sigma_{1}:=\tau_{1}-n_{1}^{2 k+1}, \sigma_{2}:=\tau_{2}-n_{2}^{2 k+1}$,

and divide the set $\left(\mathbb{R} \times \mathbb{Z}_{\lambda}^{*}\right)^{2}$ into

$$
\begin{aligned}
& A_{0}:=\left\{\left(\tau, n, \tau_{1}, n_{1}\right) \in\left(\mathbb{R} \times \mathbb{Z}_{\lambda}^{*}\right)^{2}|M=| \sigma \mid, n \neq n_{1}\right\}, \\
& A_{1}:=\left\{\left(\tau, n, \tau_{1}, n_{1}\right) \in\left(\mathbb{R} \times \mathbb{Z}_{\lambda}^{*}\right)^{2}|M=| \sigma_{1} \mid, n \neq n_{1}\right\}, \\
& A_{2}:=\left\{\left(\tau, n, \tau_{1}, n_{1}\right) \in\left(\mathbb{R} \times \mathbb{Z}_{\lambda}^{*}\right)^{2}|M=| \sigma_{2} \mid, n \neq n_{1}\right\},
\end{aligned}
$$

where $M=M\left(\tau, n, \tau_{1}, n_{1}\right):=\max \left\{|\sigma|,\left|\sigma_{1}\right|,\left|\sigma_{2}\right|\right\}$. Since

$$
M \gtrsim\left|\sigma-\sigma_{1}-\sigma_{2}\right|=\left|(-n)^{2 k+1}+n_{1}^{2 k+1}+n_{2}^{2 k+1}\right|,
$$

we have

$$
\left|n n_{1} n_{2}\right| \lesssim M
$$

when $k=1$ by Lemma 2.2 and

$$
|n|^{1-k / 2} \lesssim \lambda^{k / 2-1},\left|n_{1}\right|^{k / 2} \lesssim(\lambda M)^{1 / 4},\left|n_{2}\right|^{k / 2} \lesssim(\lambda M)^{1 / 4}
$$

when $k \geq 2$ by $\lambda^{-1} \leq|n|$ for all $n \in \mathbb{Z}_{\lambda}^{*}$ and Lemma 2.3. Thus we have

$$
|n|^{1-k / 2}\left|n_{1}\right|^{k / 2}\left|n_{2}\right|^{k / 2} \lesssim \lambda^{(k-1) / 2} M^{1 / 2}
$$

for any $k \in \mathbb{N}$. We define $\chi_{\Omega}$ as the characteristic function of a set $\Omega$.

Step 1. (Estimate for the norm $\|\cdot\|_{X^{-k / 2}(\lambda)}$ )

We prove the estimate

$$
\left\|\mathcal{F}^{-1}\left[\left\langle\tau-n^{2 k+1}\right\rangle^{-1} \widehat{\partial_{x}(u v)}\right]\right\|_{X^{-k / 2}(\lambda)} \lesssim \lambda^{\epsilon}\|u\|_{X^{-k / 2}(\lambda)}|| v \|_{X^{-k / 2}(\lambda)} .
$$


for some $\epsilon$ satisfying $0<\epsilon<(3 k-1) / 2$. From (3.1), we have

$$
\begin{aligned}
& \left\|\mathcal{F}^{-1}\left[\left\langle\tau-n^{2 k+1}\right\rangle^{-1} \widetilde{\partial_{x}(u v)}\right]\right\|_{X}^{-k / 2}(\lambda) \\
& \quad=\left\|\int_{\mathbb{Z}_{\lambda}^{*} \backslash\{n\}} \int_{\mathbb{R}} \frac{|n|^{1-k / 2}\left|n_{1}\right|^{k / 2}\left|n_{2}\right|^{k / 2}}{\langle\sigma\rangle^{1 / 2}\left\langle\sigma_{1}\right\rangle^{1 / 2}\left\langle\sigma_{2}\right\rangle^{1 / 2}} \widetilde{f}\left(\tau_{1}, n_{1}\right) \widetilde{g}\left(\tau_{2}, n_{2}\right) d \tau_{1} d n_{1}\right\|_{L_{n}^{2}(\lambda) L_{\tau}^{2}} \\
& \quad \lesssim \sum_{j=0}^{2}\left\|J_{j}\right\|_{L_{n}^{2}(\lambda) L_{\tau}^{2}},
\end{aligned}
$$

where

$$
J_{j}=\int_{\mathbb{Z}_{\lambda}^{*}} \int_{\mathbb{R}} \frac{\lambda^{(k-1) / 2} M^{1 / 2} \chi_{A_{j}}}{\langle\sigma\rangle^{1 / 2}\left\langle\sigma_{1}\right\rangle^{1 / 2}\left\langle\sigma_{2}\right\rangle^{1 / 2}} \widetilde{f}\left(\tau_{1}, n_{1}\right) \widetilde{g}\left(\tau_{2}, n_{2}\right) d \tau_{1} d n_{1} .
$$

By symmetry, we only need to consider the estimate for $J_{0}$ and $J_{1}$.

Estimate for $J_{0}$

By the Cauchy-Schwartz inequality, we have

$$
\begin{aligned}
\left\|J_{0}\right\|_{L_{n}^{2}(\lambda) L_{\tau}^{2}} & \leq \sup _{n, \sigma}\left(K_{0}\right)^{1 / 2}\|\tilde{f}\|_{L_{n}^{2}(\lambda) L_{\tau}^{2}}\|\widetilde{g}\|_{L_{n}^{2}(\lambda) L_{\tau}^{2}} \\
& =\sup _{n, \sigma}\left(K_{0}\right)^{1 / 2}\|u\|_{X^{-k / 2}(\lambda)}\|v\|_{X^{-k / 2}(\lambda)},
\end{aligned}
$$

where

$$
K_{0}=\int_{\mathbb{Z}_{\lambda}^{*}} \int_{\mathbb{R}} \frac{\lambda^{k-1} M \chi_{A_{0}}}{\langle\sigma\rangle\left\langle\sigma_{1}\right\rangle\left\langle\sigma_{2}\right\rangle} d \sigma_{1} d n_{1} \sim \int_{\mathbb{Z}_{\lambda}^{*}} \int_{\mathbb{R}} \frac{\lambda^{k-1} \chi_{A_{0}}}{\left\langle\sigma_{1}\right\rangle\left\langle\sigma_{2}\right\rangle} d \sigma_{1} d n_{1} .
$$

Let $n$ and $\sigma$ be fixed. We define $a_{1}$ and $P_{1}\left(n_{1}\right)$ as

$$
a_{1}:=\sigma_{1}+\sigma_{2}=\sigma+n^{2 k+1}-n_{1}^{2 k+1}-n_{2}^{2 k+1}=: n P_{1}\left(n_{1}\right) .
$$

Since $P_{1}(x)$ is of the form of (2.3) with $m=2 k$, from Lemmas 2.4, 2.5 and $|n| \geq \lambda^{-1}$, we have

$$
\int_{\mathbb{Z}_{\lambda}^{*}} \int_{\mathbb{R}} \frac{1}{\left\langle\sigma_{1}\right\rangle\left\langle\sigma_{2}\right\rangle} d \sigma_{1} d n_{1} \lesssim \int_{\mathbb{Z}_{\lambda}^{*}} \frac{1}{\left\langle a_{1}\right\rangle^{\delta}} d n_{1} \leq \int_{\mathbb{Z}_{\lambda}^{*}} \frac{1}{\left\langle\lambda^{-1} P_{1}\left(n_{1}\right)\right\rangle^{\delta}} d n_{1} \lesssim \lambda^{\delta(2 k+1)-1}
$$

for any $\delta$ satisfying $1 / 2 k<\delta<1$. This implies

$$
K_{0} \lesssim \lambda^{\delta(2 k+1)+k-2} \text {. }
$$

Therefore, we obtain

$$
\left\|J_{0}\right\|_{L_{n}^{2}(\lambda) L_{\tau}^{2}} \lesssim \lambda^{\delta(k+1 / 2)+k / 2-1}|| u\left\|_{X^{-k / 2}(\lambda)}\right\| v \|_{X^{-k / 2}(\lambda)}
$$

by $(3.3)$.

Estimate for $J_{1}$

By using the duality and the Cauchy-Schwartz inequality twice, we have

$$
\begin{aligned}
\left\|J_{1}\right\|_{L_{n}^{2}(\lambda) L_{\tau}^{2}} & \lesssim \sup _{n_{1}, \sigma_{1}}\left(K_{1}\right)^{1 / 2}\|\widetilde{f}\|_{L_{n_{1}}^{2}(\lambda) L_{\tau_{1}}^{2}}\|\widetilde{g}\|_{L_{n_{1}}^{2}(\lambda) L_{\tau_{1}}^{2}} \\
& =\sup _{n_{1}, \sigma_{1}}\left(K_{1}\right)^{1 / 2}\|u\|_{X^{-k / 2}(\lambda)}\|v\|_{X^{-k / 2}(\lambda)},
\end{aligned}
$$


where

$$
K_{1}=\int_{\mathbb{Z}_{\lambda}^{*}} \int_{\mathbb{R}} \frac{\lambda^{k-1} M \chi_{A_{1}}}{\langle\sigma\rangle\left\langle\sigma_{1}\right\rangle\left\langle\sigma_{2}\right\rangle} d \sigma d n \sim \int_{\mathbb{Z}_{\lambda}^{*}} \int_{\mathbb{R}} \frac{\lambda^{k-1} \chi_{A_{1}}}{\langle\sigma\rangle\left\langle\sigma_{2}\right\rangle} d \sigma d n .
$$

Let $n_{1}$ and $\sigma_{1}$ be fixed. We define $a$ and $P(n)$ as

$$
a:=\sigma-\sigma_{2}=\sigma_{1}-n^{2 k+1}+n_{1}^{2 k+1}+n_{2}^{2 k+1}=: n_{1} P(n) .
$$

Since $P(x)$ is of the form of (2.3) with $m=2 k$, from Lemmas 2.4, 2.5 and $\left|n_{1}\right| \geq \lambda^{-1}$, we have

$$
\int_{\mathbb{Z}_{\lambda}^{*}} \int_{\mathbb{R}} \frac{1}{\langle\sigma\rangle\left\langle\sigma_{2}\right\rangle} d \sigma d n \lesssim \int_{\mathbb{Z}_{\lambda}^{*}} \frac{1}{\langle a\rangle^{\delta}} d n \leq \int_{\mathbb{Z}_{\lambda}^{*}} \frac{1}{\left\langle\lambda^{-1} P(n)\right\rangle^{\delta}} d n \lesssim \lambda^{\delta(2 k+1)-1}
$$

for any $\delta$ satisfying $1 / 2 k<\delta<1$. This implies

$$
K_{1} \lesssim \lambda^{\delta(2 k+1)+k-2} \text {. }
$$

Therefore, we obtain

$$
\left\|J_{1}\right\|_{L_{n}^{2}(\lambda) L_{\tau}^{2}} \lesssim \lambda^{\delta(k+1 / 2)+k / 2-1}\|u\|_{X^{-k / 2}(\lambda)}\|v\|_{X^{-k / 2}(\lambda)}
$$

by $(3.5)$.

Putting $\epsilon:=\delta(k+1 / 2)+k / 2-1$, we obtain the estimate (3.2) by (3.4) and (3.6), where $\epsilon$ satisfies $0<\epsilon<(3 k-1) / 2$ since $1 / 2 k<\delta<1$.

Step 2. (Estimate for the norm $\|\cdot\|_{Y^{-k / 2}}$ )

We prove the estimate

$$
\left\|\mathcal{F}^{-1}\left[\left\langle\tau-n^{2 k+1}\right\rangle^{-1} \widehat{\partial_{x}(u v)}\right]\right\|_{Y^{-k / 2}(\lambda)} \lesssim \lambda^{\epsilon}\|u\|_{X^{-k / 2}(\lambda)}\|v\|_{X^{-k / 2}(\lambda)}
$$

for some $\epsilon$ satisfying $0<\epsilon<(3 k-1) / 2$. From (3.1), we have

$$
\begin{aligned}
& \left\|\mathcal{F}^{-1}\left[\left\langle\tau-n^{2 k+1}\right\rangle^{-1} \widetilde{\partial_{x}(u v)}\right]\right\|_{Y^{-k / 2}(\lambda)} \\
& =\left\|\int_{\mathbb{Z}_{\lambda}^{*} \backslash\{n\}} \int_{\mathbb{R}} \frac{|n|^{1-k / 2}\left|n_{1}\right|^{k / 2}\left|n_{2}\right|^{k / 2}}{\langle\sigma\rangle\left\langle\sigma_{1}\right\rangle^{1 / 2}\left\langle\sigma_{2}\right\rangle^{1 / 2}} \tilde{f}\left(\tau_{1}, n_{1}\right) \widetilde{g}\left(\tau_{2}, n_{2}\right) d \tau_{1} d n_{1}\right\|_{L_{n}^{2}(\lambda) L_{\tau}^{1}} \\
& \quad \lesssim \sum_{j=0}^{2}\left\|I_{j}\right\|_{L_{n}^{2}(\lambda)},
\end{aligned}
$$

where

$$
I_{j}=\int_{\mathbb{R}} \int_{\mathbb{Z}_{\lambda}^{*}} \int_{\mathbb{R}} \frac{\lambda^{(k-1) / 2} M^{1 / 2} \chi_{A_{j}}}{\langle\sigma\rangle\left\langle\sigma_{1}\right\rangle^{1 / 2}\left\langle\sigma_{2}\right\rangle^{1 / 2}}\left|\tilde{f}\left(\tau_{1}, n_{1}\right)\right|\left|\widetilde{g}\left(\tau_{2}, n_{2}\right)\right| d \tau_{1} d n_{1} d \tau .
$$

By symmetry, we only need to consider the estimate for $I_{0}$ and $I_{1}$.

Estimate for $I_{0}$

By the Cauchy-Schwartz inequality, we have

$$
\begin{aligned}
\left\|I_{0}\right\|_{L_{n}^{2}(\lambda)} & \leq \sup _{n}\left(L_{0}\right)^{1 / 2}\|\widetilde{f}\|_{L_{n}^{2}(\lambda) L_{\tau}^{2}}\|\widetilde{g}\|_{L_{n}^{2}(\lambda) L_{\tau}^{2}} \\
& =\sup _{n}\left(L_{0}\right)^{1 / 2}\|u\|_{X^{-k / 2}(\lambda)}\|v\|_{X^{-k / 2}(\lambda)},
\end{aligned}
$$


where

$$
L_{0}=\int_{\mathbb{R}} \int_{\mathbb{Z}_{\lambda}^{*}} \int_{\mathbb{R}} \frac{\lambda^{k-1} M \chi_{A_{0}}}{\langle\sigma\rangle^{2}\left\langle\sigma_{1}\right\rangle\left\langle\sigma_{2}\right\rangle} d \sigma_{1} d n_{1} d \sigma \sim \int_{\mathbb{R}} \int_{\mathbb{Z}_{\lambda}^{*}} \int_{\mathbb{R}} \frac{\lambda^{k-1} \chi_{A_{0}}}{\langle\sigma\rangle\left\langle\sigma_{1}\right\rangle\left\langle\sigma_{2}\right\rangle} d \sigma_{1} d n_{1} d \sigma .
$$

Let $n$ be fixed and we define $a_{1}$ and $P_{1}\left(n_{1}\right)$ as

$$
a_{1}:=-n^{2 k+1}+n_{1}^{2 k+1}+n_{2}^{2 k+1}=: n P_{1}\left(n_{1}\right) \text {. }
$$

Since $\sigma_{2}=\sigma-\left(\sigma_{1}+a_{1}\right)$ and $P_{1}(x)$ is of the form of (2.3) with $m=2 k$, from Lemmas 2.4, 2.5 and $|n| \geq \lambda^{-1}$, we have

$$
\begin{gathered}
\int_{\mathbb{Z}_{\lambda}^{*}} \int_{\mathbb{R}}\left(\int_{\mathbb{R}} \frac{1}{\langle\sigma\rangle\left\langle\sigma_{2}\right\rangle} d \sigma\right) \frac{1}{\left\langle\sigma_{1}\right\rangle} d \sigma_{1} d n_{1} \lesssim \int_{\mathbb{Z}_{\lambda}^{*}} \int_{\mathbb{R}} \frac{1}{\left\langle\sigma_{1}+a_{1}\right\rangle^{1-\delta^{\prime}}\left\langle\sigma_{1}\right\rangle} d \sigma_{1} d n_{1} \\
\quad \lesssim \int_{\mathbb{Z}_{\lambda}^{*}} \frac{1}{\left\langle a_{1}\right\rangle^{\delta-\delta^{\prime}}} d n_{1} \leq \int_{\mathbb{Z}_{\lambda}^{*}} \frac{1}{\left\langle\lambda^{-1} P_{1}\left(n_{1}\right)\right\rangle^{\delta-\delta^{\prime}}} d n_{1} \lesssim \lambda^{\left(\delta-\delta^{\prime}\right)(2 k+1)-1}
\end{gathered}
$$

for any $\delta$ and $\delta^{\prime}$ satisfying $0<\delta^{\prime}<\delta<1$ and $1 / 2 k<\delta-\delta^{\prime}$. This implies

$$
L_{0} \lesssim \lambda^{\left(\delta-\delta^{\prime}\right)(2 k+1)+k-2} .
$$

Therefore, we obtain

$$
\left\|I_{0}\right\|_{L_{n}^{2}(\lambda)} \lesssim \lambda^{\left(\delta-\delta^{\prime}\right)(k+1 / 2)+k / 2-1}\|u\|_{X^{-k / 2}(\lambda)}\|v\|_{X^{-k / 2}(\lambda)}
$$

by $(3.8)$.

\section{Estimate for $I_{1}$}

Let $\gamma>0$. By the Cauchy-Schwartz inequality, we have

$$
\left\|I_{1}\right\|_{L_{n}^{2}(\lambda)} \lesssim\left\|\int _ { \mathbb { Z } _ { \lambda } ^ { * } } \int _ { \mathbb { R } } \frac { \lambda ^ { ( k - 1 ) / 2 } M ^ { 1 / 2 } \chi _ { A _ { 1 } } } { \langle \sigma \rangle ^ { 1 / 2 - \gamma } \langle \sigma _ { 1 } \rangle ^ { 1 / 2 } \langle \sigma _ { 2 } \rangle ^ { 1 / 2 } } \left|\tilde{f}\left(\tau_{1}, n_{1}\right)\left\|\widetilde{g}\left(\tau_{2}, n_{2}\right) \mid d \tau_{1} d n_{1}\right\|_{L_{n}^{2}(\lambda) L_{\tau}^{2}} .\right.\right.
$$

By using the duality and the Cauchy-Schwartz inequality twice, we have

$$
\begin{aligned}
\left\|I_{1}\right\|_{L_{n}^{2}(\lambda)} & \lesssim \sup _{n_{1}, \sigma_{1}}\left(L_{1}\right)^{1 / 2}\|\widetilde{f}\|_{L_{n_{1}}^{2}(\lambda) L_{\tau_{1}}^{2}}\|\widetilde{g}\|_{L_{n_{1}}^{2}(\lambda) L_{\tau_{1}}^{2}} \\
& =\sup _{n_{1}, \sigma_{1}}\left(L_{1}\right)^{1 / 2}\|u\|_{X^{-k / 2}(\lambda)}\|v\|_{X^{-k / 2}(\lambda)},
\end{aligned}
$$

where

$$
L_{1}=\int_{\mathbb{Z}_{\lambda}^{*}} \int_{\mathbb{R}} \frac{\lambda^{k-1} M \chi_{A_{1}}}{\langle\sigma\rangle^{1-2 \gamma}\left\langle\sigma_{1}\right\rangle\left\langle\sigma_{2}\right\rangle} d \sigma d n \sim \int_{\mathbb{Z}_{\lambda}^{*}} \int_{\mathbb{R}} \frac{\lambda^{k-1} \chi_{A_{1}}}{\langle\sigma\rangle^{1-2 \gamma}\left\langle\sigma_{2}\right\rangle} d \sigma d n .
$$

Let $n_{1}$ and $\sigma_{1}$ be fixed. We define $a$ and $P(n)$ as

$$
a:=\sigma-\sigma_{2}=\sigma_{1}-n^{2 k+1}+n_{1}^{2 k+1}+n_{2}^{2 k+1}=: n_{1} P(n) .
$$

Since $P(x)$ is of the form of (2.3) with $m=2 k$, from Lemmas 2.4, 2.5 and $\left|n_{1}\right| \geq \lambda^{-1}$, we have

$$
\begin{aligned}
\int_{\mathbb{Z}_{\lambda}^{*}} \int_{\mathbb{R}} \frac{1}{\langle\sigma\rangle^{1-2 \gamma}\left\langle\sigma_{2}\right\rangle} d \sigma d n & \lesssim \int_{\mathbb{Z}_{\lambda}^{*}} \frac{1}{\langle a\rangle^{\delta-2 \gamma}} d n \leq \int_{\mathbb{Z}_{\lambda}^{*}} \frac{1}{\left\langle\lambda^{-1} P(n)\right\rangle^{\delta-2 \gamma}} d n \\
& \lesssim \lambda^{(\delta-2 \gamma)(2 k+1)-1}
\end{aligned}
$$

for all $\delta$ satisfying $1 / 2 k<\delta-2 \gamma<1$. This implies

$$
L_{1} \lesssim \lambda^{(\delta-2 \gamma)(2 k+1)+k-2} \text {. }
$$


Therefore, we obtain

$$
\left\|I_{1}\right\|_{L_{n}^{2}(\lambda)} \lesssim \lambda^{(\delta-2 \gamma)(k+1 / 2)+k / 2-1}\|u\|_{X^{-k / 2}(\lambda)}\|v\|_{X^{-k / 2}(\lambda)}
$$

by $(3.10)$.

Putting $\gamma=\delta^{\prime} / 2$ and $\epsilon:=\left(\delta-\delta^{\prime}\right)(k+1 / 2)+k / 2-1$, we obtain the estimate (3.7) by (3.9) and (3.11), where $\epsilon$ satisfies $0<\epsilon<(3 k-1) / 2$ since $1 / 2 k<\delta-\delta^{\prime}<1$.

Next, we prove Theorem 1.4.

Proof of Theorem 1.4. Let $N \gg 1$, and we put

$$
\begin{aligned}
& \widetilde{u}_{N}(\tau, n):=\left(\chi_{\{N\}}(n)+\chi_{\{N\}}(-n)\right) \chi_{[-1,1]}\left(\tau-n^{2 k+1}\right), \\
& \widetilde{v}_{N}(\tau, n):=\left(\chi_{\{1-N\}}(n)+\chi_{\{1-N\}}(-n)\right) \chi_{[-1,1]}\left(\tau-n^{2 k+1}\right),
\end{aligned}
$$

where

$$
\chi_{\{c\}}(n):=\left\{\begin{array}{l}
1(n=c) \\
0(n \neq c)
\end{array}, \chi_{[-1,1]}(\sigma):=\left\{\begin{array}{l}
1(|\sigma| \leq 1) \\
0(|\sigma|>1)
\end{array} .\right.\right.
$$

for $c \in \mathbb{Z}$. Then we have

$$
\left\|u_{N}\right\|_{Z^{s}} \sim\left\|v_{N}\right\|_{Z^{s}} \sim N^{s}\left(\left\|\chi_{[-1,1]}\right\|_{L_{\sigma}^{2}}+\left\|\chi_{[-1,1]}\right\|_{L_{\sigma}^{1}}\right) \sim N^{s} .
$$

We put

$$
\begin{aligned}
& A_{1}\left(n_{1}, n_{2}\right):=\chi_{\{N\}}\left(n_{1}\right) \chi_{\{1-N\}}\left(n_{2}\right), A_{2}\left(n_{1}, n_{2}\right):=\chi_{\{N\}}\left(n_{1}\right) \chi_{\{1-N\}}\left(-n_{2}\right), \\
& A_{3}\left(n_{1}, n_{2}\right):=\chi_{\{N\}}\left(-n_{1}\right) \chi_{\{1-N\}}\left(n_{2}\right), A_{4}\left(n_{1}, n_{2}\right):=\chi_{\{N\}}\left(-n_{1}\right) \chi_{\{1-N\}}\left(-n_{2}\right) .
\end{aligned}
$$

Then we have

$$
\begin{aligned}
& \left.\| \mathcal{F}^{-1}\left[\left\langle\tau-n^{2 k+1}\right\rangle^{-1} \partial_{x} \widetilde{\left(u_{N} v_{N}\right.}\right)\right] \|_{X^{s}} \\
& =\left\|\sum_{j=1}^{4} \int_{\mathbb{Z}^{*}}|n|^{s+1} A_{j}\left(n_{1}, n_{2}\right)\left(\int_{\mathbb{R}}\langle\sigma\rangle^{-1 / 2} \chi_{[-1,1]}\left(\sigma_{1}\right) \chi_{[-1,1]}\left(\sigma_{2}\right) d \sigma_{1}\right) d n_{1}\right\|_{L_{n}^{2} L_{\sigma}^{2}} .
\end{aligned}
$$

From $\sigma_{2}=n^{2 k+1}-n_{1}^{2 k+1}-n_{2}^{2 k+1}+\sigma-\sigma_{1}$ and Lemma 2.2, we have

$$
\langle\sigma\rangle \sim\left|n^{2 k+1}-n_{1}^{2 k+1}-n_{2}^{2 k+1}\right| \sim\left|n n_{1} n_{2}\right| \max \left\{|n|,\left|n_{1}\right|,\left|n_{2}\right|\right\}^{2 k-2}
$$

when there exists $\sigma_{1}$ satisfying $\left|\sigma_{1}\right| \leq 1$ and $\left|\sigma_{2}\right| \leq 1$. This implies

$$
\int_{\mathbb{R}}\langle\sigma\rangle^{-1 / 2} \chi_{[-1,1]}\left(\sigma_{1}\right) \chi_{[-1,1]}\left(\sigma_{2}\right) d \sigma_{1} \gtrsim\left|n n_{1} n_{2}\right|^{-1 / 2} \max \left\{|n|,\left|n_{1}\right|,\left|n_{2}\right|\right\}^{-(k-1)} \text {. }
$$

As a result, we obtain

$$
\begin{aligned}
& \left.\| \mathcal{F}^{-1}\left[\left\langle\tau-n^{2 k+1}\right\rangle^{-1} \partial_{x} \widetilde{\left(u_{N} v_{N}\right.}\right)\right] \|_{X^{s}} \\
& \gtrsim\left\|\sum_{j=1}^{4} \int_{\mathbb{Z}^{*}}|n|^{s+1} A_{j}\left(n_{1}, n_{2}\right)\left|n n_{1} n_{2}\right|^{-1 / 2} \max \left\{|n|,\left|n_{1}\right|,\left|n_{2}\right|\right\}^{-(k-1)} d n_{1}\right\|_{L_{n}^{2}} \\
& \sim\left\|N^{-k}\left(\chi_{\{1\}}+\chi_{\{-1\}}\right)+N^{s+1} N^{-k-1 / 2}\left(\chi_{\{2 N-1\}}+\chi_{\{-2 N+1\}}\right)\right\|_{L_{n}^{2}} \\
& \gtrsim N^{-k} .
\end{aligned}
$$


By above discussion, if the bilinear estimate (1.6) holds, then we have

$$
\begin{aligned}
N^{-k} & \lesssim\left\|\mathcal{F}^{-1}\left[\left\langle\tau-n^{2 k+1}\right\rangle^{-1} \partial_{x} \widetilde{\left(u_{N} v_{N}\right)}\right]\right\|_{X^{s}} \leq\left\|\mathcal{F}^{-1}\left[\left\langle\tau-n^{2 k+1}\right\rangle^{-1} \partial_{x} \widetilde{\left(u_{N} v_{N}\right)}\right]\right\|_{Z^{s}} \\
& \lesssim\left\|u_{N}\right\|_{Z^{s}}\left\|v_{N}\right\|_{Z^{s}} \sim N^{2 s},
\end{aligned}
$$

which contradicts the assumption $s<-k / 2$.

\section{Proof of local well-posedness}

In this section, we prove LWP of (1.1) by using the bilinear estimate. First, we consider the scaling property. (1.1) with initial data $u_{0} \in \widetilde{H}^{s}(\mathbb{T})$ is invariant under the following scaling;

$$
u_{\lambda}(t, x)=\lambda^{-2 k} u\left(\lambda^{-2 k-1} t, \lambda^{-1} x\right), \quad u_{\lambda 0}(x)=\lambda^{-2 k} u_{0}\left(\lambda^{-1} x\right) .
$$

More precisely, if $u$ satisfies $(1.1)$ on $[0, T] \times \mathbb{T}$ with initial data $u_{0} \in \widetilde{H}^{s}(\mathbb{T})$, then $u_{\lambda}$ satisfies the same equation on $\left[0, \lambda^{2 k+1} T\right] \times \mathbb{T}_{\lambda}$ with initial data $u_{\lambda 0} \in$ $\widetilde{H}^{s}\left(\mathbb{T}_{\lambda}\right)$.

Proposition 4.1. For $s \in \mathbb{R}$, and $\lambda \geq 1$, we have

$$
\left\|u_{\lambda 0}\right\|_{\widetilde{H}^{s}\left(\mathbb{T}_{\lambda}\right)}=\lambda^{-2 k-s+1 / 2}\left\|u_{0}\right\|_{\widetilde{H}^{s}(\mathbb{T})},
$$

where

$$
\|\left. f\right|_{\widetilde{H}^{s}\left(\mathbb{T}_{\lambda}\right)}:=\left.\left.|| n\right|^{s} \widehat{f}\right|_{L_{n}^{2}(\lambda)}
$$

Proof. We note that

$$
\left(\frac{1}{2 \pi \lambda} \sum_{n \in \mathbb{Z}_{\lambda}^{*}}|f(\lambda n)|^{2}\right)^{1 / 2}=\lambda^{-1 / 2}\left(\frac{1}{2 \pi} \sum_{n \in \mathbb{Z}^{*}}|f(n)|^{2}\right)^{1 / 2}
$$

for all function $f$ defined on $\mathbb{T}$. This implies

$$
\|f(\lambda n)\|_{L_{n}^{2}(\lambda)}=\lambda^{-1 / 2}\|f\|_{L_{n}^{2}} .
$$

Since $\widehat{u}_{\lambda 0}(n)=\lambda^{-2 k+1} \widehat{u}_{0}(\lambda n)$ and

$$
|||n|^{s} \lambda^{-2 k+1} \widehat{u}_{0}(\lambda n)\left\|_{L_{n}^{2}(\lambda)}=\lambda^{-1 / 2}||\left|\lambda^{-1} n\right|^{s} \lambda^{-2 k+1} \widehat{u}_{0}\right\|_{L_{n}^{2}}
$$

by (4.3), we obtain (4.2).

Next, we give the linear estimates for the Eq. (1.1).

Proposition 4.2. Let $\lambda \geq 1$. We put

$$
\left(U_{\lambda}(t) f\right)(x):=\int_{\mathbb{Z}_{\lambda}^{*}} \exp \left(i t n^{2 k+1}+i x n\right) \widehat{f}(n) d n
$$

and

$$
\left(U_{\lambda} *_{R} F\right)(t):=\int_{0}^{t} U_{\lambda}\left(t-t^{\prime}\right) F\left(t^{\prime}\right) d t^{\prime}
$$

For $s \in \mathbb{R}$, there exist positive constants $C_{1}$ and $C_{2}$ such that

$$
\left\|\psi U_{\lambda}(\cdot) f\right\|_{Z^{s}(\lambda)} \leq C_{1}\|f\|_{\widetilde{H}^{s}\left(\mathbb{T}_{\lambda}\right)},
$$




$$
\left\|\psi\left(U_{\lambda} *_{R} F\right)\right\|_{Z^{s}(\lambda)} \leq C_{2}\left\|\mathcal{F}^{-1}\left[\left\langle\tau-n^{2 k+1}\right\rangle^{-1} \widetilde{F}\right]\right\|_{Z^{s}(\lambda)},
$$

where $C_{1}$ and $C_{2}$ do not depend on $\lambda$ and $\psi$ is a cut-off function such that $\psi \in C^{\infty}(\mathbb{R}), \psi(t)=1$ on $[-1,1]$ and $\operatorname{supp} \psi \subset[-2,2]$.

For the proof of Proposition 4.2, see Lemma 2.1 in [8].

Proof of Theorem 1.2. For $u_{0} \in B_{r}\left(\widetilde{H}^{s}(\mathbb{T})\right)$, we choose $\lambda>1$ such that $\lambda^{2 k+s-1 / 2-\epsilon} \geq 4 C_{0} C_{1} C_{2} r$, where $C_{1}$ and $C_{2}$ appeared in Proposition 4.2, and $C_{0}$ and $\epsilon$ appeared in Theorem 1.3. Then we have $\left\|u_{\lambda 0}\right\|_{\widetilde{H}^{s}\left(\mathbb{T}_{\lambda}\right)} \leq$ $1 /\left(4 C_{0} C_{1} C_{2} \lambda^{\epsilon}\right)=: R_{\lambda}$ from Proposition 4.1. Therefore, we assume that $u_{\lambda 0} \in B_{R_{\lambda}}\left(\widetilde{H}^{s}\left(\mathbb{T}_{\lambda}\right)\right)$.

We define the map $\Phi_{u_{0}, \lambda}$ as

$$
\Phi_{u_{0}, \lambda}[u](t):=\psi(t) U_{\lambda}(t) u_{\lambda 0}-\frac{\psi(t)}{2} \int_{0}^{t} U_{\lambda}\left(t-t^{\prime}\right) \partial_{x}\left(u\left(t^{\prime}\right)^{2}\right) d t^{\prime},
$$

where $\psi$ is a cut function satisfying the assumption in Proposition 4.2. To prove the existence of the solution of (1.1), we first prove that $\Phi_{u_{0}, \lambda}$ is a contraction map on $B_{2 C_{1} R_{\lambda}}\left(Z^{s}(\lambda)\right)$.

Step 1. (Existence)

For any $u \in B_{2 C_{1} R_{\lambda}}\left(Z^{s}(\lambda)\right)$, from Proposition 4.2 and Theorem 1.3, we have

$$
\begin{aligned}
\left\|\Phi_{u_{0}, \lambda}[u]\right\|_{Z^{s}(\lambda)} & \leq\left\|\psi U_{\lambda}(\cdot) u_{\lambda 0}\right\|_{Z^{s}(\lambda)}+\frac{1}{2}\left\|\psi\left(U_{\lambda} *_{R}\left(\partial_{x}\left(u^{2}\right)\right)\right)\right\|_{Z^{s}(\lambda)} \\
& \leq C_{1} R_{\lambda}+\frac{1}{2} C_{2} C_{0} \lambda^{\epsilon}\|u\|_{Z^{s}(\lambda)}^{2} \\
& \leq C_{1} R_{\lambda}+\left(2 C_{0} C_{1} C_{2} \lambda^{\epsilon} R_{\lambda}\right) C_{1} R_{\lambda} \leq 2 C_{1} R_{\lambda} .
\end{aligned}
$$

Therefore, we obtain $\Phi_{u_{0}, \lambda}[u] \in B_{2 C_{1} R_{\lambda}}\left(Z^{s}(\lambda)\right)$.

For any $u, v \in B_{2 C_{1} R_{\lambda}}\left(Z^{s}(\lambda)\right)$, from Proposition 4.2 and Theorem 1.3, we have

$$
\begin{aligned}
\left\|\Phi_{u_{0}, \lambda}[u]-\Phi_{u_{0}, \lambda}[v]\right\|_{Z^{s}(\lambda)} & \leq \frac{1}{2}\left\|\psi\left(U_{\lambda} *_{R}\left(\partial_{x}\left(u^{2}-v^{2}\right)\right)\right)\right\|_{Z^{s}(\lambda)} \\
& \leq \frac{1}{2} C_{2} C_{0} \lambda^{\epsilon}\|u+v\|_{Z^{s}(\lambda)}\|u-v\|_{Z^{s}(\lambda)} \\
& \leq 2 C_{0} C_{1} C_{2} \lambda^{\epsilon} R_{\lambda}\|u-v\|_{Z^{s}(\lambda)}=\frac{1}{2}\|u-v\|_{Z^{s}(\lambda)} .
\end{aligned}
$$

This implies that $\Phi_{u_{0}, \lambda}$ is a contraction map.

By above discussion and applying Banach's fixed point theorem, there exists $u_{\lambda}^{\prime} \in B_{2 C_{1} R_{\lambda}}\left(Z^{s}(\lambda)\right)$, such that

$$
u_{\lambda}^{\prime}(t)=\psi(t) U_{\lambda}(t) u_{\lambda 0}-\frac{\psi(t)}{2} \int_{0}^{t} U_{\lambda}\left(t-t^{\prime}\right) \partial_{x}\left(u_{\lambda}^{\prime}\left(t^{\prime}\right)^{2}\right) d t^{\prime} .
$$

Especially, $u_{\lambda}^{\prime}$ satisfies the integral equation

$$
u_{\lambda}^{\prime}(t)=U_{\lambda}(t) u_{\lambda 0}-\frac{1}{2} \int_{0}^{t} U_{\lambda}\left(t-t^{\prime}\right) \partial_{x}\left(u_{\lambda}^{\prime}\left(t^{\prime}\right)^{2}\right) d t^{\prime} .
$$


in the time interval $[0,1]$. Therefore, $u_{\lambda}=\left.u_{\lambda}^{\prime}\right|_{[0,1]} \in B_{2 C_{1} R_{\lambda}}\left(Z_{[0,1]}^{s}(\lambda)\right)$ is the time local solution of (1.1).

Next, we prove that $u_{\lambda}$ is unique in $B_{2 C_{1} R_{\lambda}}\left(Z_{[0,1]}^{s}(\lambda)\right)$.

Step 2. (Uniqueness)

We assume that $u_{\lambda}, v_{\lambda} \in B_{2 C_{1} R_{\lambda}}\left(Z_{[0,1]}^{s}(\lambda)\right)$ satisfy (4.8) on $[0,1]$. For any $u_{\lambda}^{\prime}$, $v_{\lambda}^{\prime} \in Z^{s, 1 / 2}(\lambda)$ such that $u_{\lambda}^{\prime}(t)=u_{\lambda}(t), v_{\lambda}^{\prime}(t)=v_{\lambda}(t)$ on $[0,1]$, we have

$$
\begin{aligned}
\left\|u_{\lambda}-v_{\lambda}\right\|_{Z_{[0,1]}^{s}(\lambda)} & \leq \frac{1}{2}\left\|\psi\left(U_{\lambda} *_{R}\left(\partial_{x}\left(u_{\lambda}^{2}-v_{\lambda}^{2}\right)\right)\right)\right\|_{Z_{[0,1]}^{s}(\lambda)} \\
& \leq \frac{1}{2}\left\|\psi\left(U_{\lambda} *_{R}\left(\partial_{x}\left(\left(u_{\lambda}^{\prime}\right)^{2}-\left(v_{\lambda}^{\prime}\right)^{2}\right)\right)\right)\right\|_{Z^{s}(\lambda)} \\
& \leq \frac{1}{2} C_{2} C_{0} \lambda^{\epsilon}\left\|u_{\lambda}^{\prime}+v_{\lambda}^{\prime}\right\|_{Z^{s}(\lambda)}\left\|u_{\lambda}^{\prime}-v_{\lambda}^{\prime}\right\|_{Z^{s}(\lambda)}
\end{aligned}
$$

from Proposition 4.2 and Theorem 1.3. Therefore we obtain

$$
\begin{aligned}
\left\|u_{\lambda}-v_{\lambda}\right\|_{Z_{[0,1]}^{s}(\lambda)} & \leq \frac{1}{2} C_{2} C_{0} \lambda^{\epsilon}\left\|u_{\lambda}+v_{\lambda}\right\|_{Z_{[0,1]}^{s}(\lambda)}\left\|u_{\lambda}-v_{\lambda}\right\|_{Z_{[0,1]}^{s}(\lambda)} \\
& \leq 2 C_{0} C_{1} C_{2} \lambda^{\epsilon} R_{\lambda}\left\|u_{\lambda}-v_{\lambda}\right\|_{Z_{[0,1]}^{s}(\lambda)} \\
& =\frac{1}{2}\left\|u_{\lambda}-v_{\lambda}\right\|_{Z_{[0,1]}^{s}(\lambda)} .
\end{aligned}
$$

Thus, $u_{\lambda}(t)=v_{\lambda}(t)$ on $[0,1]$.

Finally, we prove that the data-to-solution map is Lipschitz continuous.

Step 3. (Lipschitz continuity of the data-to-solution map)

Let $u_{\lambda}^{\prime}$ (resp. $\left.v_{\lambda}^{\prime}\right) \in B_{2 C_{1} R_{\lambda}}\left(Z^{s, 1 / 2}(\lambda)\right)$ be the solution of (4.7) with initial data $u_{\lambda 0}$ (resp. $\left.v_{\lambda 0}\right) \in B_{R_{\lambda}}\left(\widetilde{H}^{s}\left(\mathbb{T}_{\lambda}\right)\right)$ obtained in Step 1. Then we have

$$
\left\|u_{\lambda}^{\prime}-v_{\lambda}^{\prime}\right\|_{Z^{s}(\lambda)} \leq C_{1}\left\|u_{\lambda 0}-v_{\lambda 0}\right\|_{\widetilde{H}^{s}\left(\mathbb{T}_{\lambda}\right)}+\frac{1}{2}\left\|u_{\lambda}^{\prime}-v_{\lambda}^{\prime}\right\|_{Z^{s}(\lambda)} .
$$

in the same manner as above. Therefore, we obtain

$$
\left\|u_{\lambda}^{\prime}-v_{\lambda}^{\prime}\right\|_{Z^{s}(\lambda)} \leq 2 C_{1}\left\|u_{\lambda 0}-v_{\lambda 0}\right\|_{\widetilde{H}^{s}\left(\mathbb{T}_{\lambda}\right)} .
$$

This implies that the data-to-solution map is Lipschitz continuous.

\section{Acknowledgments}

The author would like to express his appreciation to Kotaro Tsugawa for many discussions and very valuable comments and Nobu Kishimoto for the advice about Lemma 2.2.

\section{References}

[1] Bona, J., Smith, R.: The initial-value problem for the Korteweg-de Vries equation. Roy. Soc. Lond. Ser. A 278, 555-601 (1975)

[2] Bourgain, J.: Fourier transform restriction phenomena for certain lattice subsets and applications to nonlinear evolution equations, I, II. Geom. Funct. Anal. 3, 107-156, 209-262 (1993) 
[3] Chen, W., Li, J., Miao, C., Wu, J.: Low regularity solution of two fifth-order KdV type equations. J. D'Anal. Math. 107, 221-238 (2009)

[4] Christ, M., Colliander, J., Tao, T.: Asymptotics, frequency modulation, and low regularity ill-posedness for canonical defocusing equations. Am. J. Math. 125, 1235-1293 (2003)

[5] Colliander, J., Keel, M., Staffilani, G., Takaoka, H., Tao, T.: Sharp global wellposedness for $\mathrm{KdV}$ and modified $\mathrm{KdV}$ on $\mathbb{R}$ and $\mathbb{T}$. J. Am. Math. Soc 16, 705-749 (2003)

[6] Cui, S., Deng, D., Tao, S.: Global existence of solutions for the Cauchy problem of the Kawahara equation with $L^{2}$ initial data. Acta Math. Sin. 22, 1457-1466 (2006)

[7] Ginibre, J., Tsutsumi, Y.: Uniqueness of solutions for the generalized Kortewegde Vries equation. SIAM J. Math. Anal. 20, 1388-1425 (1989)

[8] Ginibre, J., Tsutsumi, Y., Velo, G.: On the Cauchy problem for the Zakharov system. J. Funct. Anal. 151, 384-436 (1997)

[9] Gorsky, J., Himonas, A.A.: Well-posedness of KdV with higher dispersion. Math. Comput. Simul. 80, 173-183 (2009)

[10] Guo, Z.: Global well-posedness of Korteweg-de Vries equation in $H^{-3 / 4}(\mathbb{R})$. J. Math. Pures Appl. 91, 583-597 (2009)

[11] Kato, T.: On the Korteweg-de Vries equation. Manuscripta Math. 28, 89-99 (1979)

[12] Kato, T.: Well-posedness for the fifth order $\mathrm{KdV}$ equation (preprint). arXiv:1011.3956 [math.AP]

[13] Kenig, C., Ponce, G., Vega, L.: Well-posedness of the initial value problem for the Korteweg-de Vries. J. Am. Math. Soc. 4, 323-347 (1991)

[14] Kenig, C., Ponce, G., Vega, L.: Well-posedness and scattering results for the generalized Korteweg-de Vries equation via the contraction principle. Commun. Pure Appl. Math. 46, 527-560 (1993)

[15] Kenig, C., Ponce, G., Vega, L.: The Cauchy problem for the Korteweg-de Vries equation in Sobolev spaces of negative indices. Duke Math. J. 71, 1-21 (1993)

[16] Kenig, C., Ponce, G., Vega, L.: Higher-order nonlinear dispersive equations. Commun. Pure Appl. Math. Soc. 122(1), 157-166 (1994)

[17] Kenig, C., Ponce, G., Vega, L.: A bilinear estimate with applications to the KdV equation. J. Am. Math. Soc. 9, 573-603 (1996)

[18] Kenig, G., Ponce, G., Vega, L.: On the ill-posedness of some canonical dispersive equations. Duke Math. J. 106(3), 617-633 (2001)

[19] Kishimoto, N.: Well-posedness of the Cauchy problem for the Korteweg-de Vries equation at the critical regularity. Differ. Integral Equ. 22(5-6), 447-464 (2009) 
[20] Kwon, S.: On the fifth order KdV equation : Local well-posedness and lack of uniform continuity of the solution map. J. Differ. Equ. 245(9), 2627-2659 (2008)

[21] Nakanishi, K., Takaoka, H., Tsutsumi, Y.: Counterexamples to bilinear estimates related with the $\mathrm{KdV}$ equation and the nonlinear Schrödinger equation. Methods Appl. Anal. 8, 569-578 (2001)

[22] Pilod, D.: On the Cauchy problem for higher-order nonlinear dispersive equations. J. Differ. Equ. 245(8), 2055-2077 (2008)

[23] Ponce, G.: Lax pairs and higher order models for water waves. J. Differ. Equ. 102(2), 360-381 (1993)

[24] Tsugawa, K.: Global well-posedness for the KdV equations on the real line with low regularity forcing terms. Commun. Contemp. Math. 8(5), 681-713 (2006)

[25] Wang, H., Cui, S., Deng, D.: Global existence of solution for the Kawahara equation in Sobolev spaces of negative indices. Acta Math. Sin. 23, 1435-1446 (2007)

H. Hirayama

Graduate School of Mathematics

Nagoya University

Chikusa-ku

Nagoya 464-8602

Japan

e-mail: m08035f@math.nagoya-u.ac.jp

Received: 31 August 2010.

Accepted: 3 December 2011. 